\title{
Aim and approach of this book
}

Microfluidics has proven to be of versatile utility in both academic and industrial laboratories, with still further promise. It is therefore worthwhile to consider exploration of its rich potential in a plethora of research facilities. As positive as this sounds, though, it confronts scientists and engineers with a challenge: how to get acquainted with this technique? This is where this book comes into play.

It is the aim of this textbook to give beginners a concise and convincing understanding of the physical-chemical principles of microfluidics, along with further giving a handy and helpful practical guideline to practice one's first (and second) own experiments. In Chapter 1, we detail about the fundamental physics of fluid transport, along with the basics of further areas that are relevant for microfluidics. Chapter 2 then gives an introduction to a prime branch of microchannel-based fluidics, which is that of segmented fluid streams with immiscible components (also named droplet-based microfluidics). Finally, Chapter 3 gets to the heart of this book's intent and presents practical guidelines to the assembly and operation of microfluidic devices, both for droplet-based microfluidics and for the flow of continuous fluid streams with miscible components (also named co-flow microfluidics). A special emphasis is on troubleshooting and acquiring competence for conceptual understanding about which experimental conditions will lead to which type of flow in a microchannel system.

The target group of all the matter introduced in the three main chapters of this book is diverse. As readers from different disciplines or with different intent of reading this book may have different interest in following detailed derivations of equations, in Chapter 1, each equation that is a central cornerstone of practical utility is framed, whereas those "only" serving as the way to get to these cornerstones are given without such special emphasis. With this approach, we ensure that each equation is well comprehensible and does not just appear from nowhere, whereas readers with a more pragmatic intention may skip these parts and just consider the framed mathematical milestones.

In the Preface section, it is stated that "there is no standard in education and training on this method [meaning: in microfluidics]". Yet, there is fantastic standards in the education and training on the fundamental physics that lays the fundament for this method. Part of that standard is outlined in Chapter 1 of this book, partially following and inspired by the didactical lines of the textbooks "Experimentalphysik 1" by W. Demtröder (Springer, 1998), "Physikalische Chemie" by P. W. Atkins (Wiley, 1996), and "Polymer Physics" by M. Rubinstein and R. H. Colby (Oxford University Press, 2003), which are the authors' sources of knowledge on elementary fluid dynamics, molecular motion, and polymer viscoelasticity. 
